cant concentrations of partially oxygenated species, which are inferred to be in some wise different from the pure $\mathrm{T}$ and $\mathrm{R}$ states. Taken at face value this would seem to add a new degree of complexity in the system.

This is perhaps a good moment to relate that my account some time ago (Nature, 237, 481; 1972) of the work of Perutz and his colleagues on the structural and functional properties of ferrous-ferric hybrid haemoglobins occasioned a frightful eruption, and a rain of shrapnel. An irate reader wrote to state that Perutz had made a rudimentary error in his assignments of high and low-spin spectroscopic features in the visible, and had in other respects abused his data, and I was charged with perpetuating if not amplifying his errors. Having taken it all on the chin, I will not tax readers with the details: the model as outlined is now hanging fire, while Perutz prepares new arguments, which are promised in detail soon.

\section{POPULATION CYCLES Why Study Dispersal?}

from a Correspondent

Population cycles of abundance of small rodents have kindled interest in ecologists for almost half a century, and several theories purporting to explain them have been put forward. All these theories have been rather less than convincing because they lacked reinforcement from experiments in the field. Some interesting observations on rodent cycles are now put forward by Krebs, Gaines, Keller, Myers and Tamarin (Science, 179, 35; 1973).

Krebs et al. started with the ideas put forward by D. Chitty (Proc. Ecol. Soc. Aust., 2,51 ; 1967) that the genetic composition of the population changes during a cycle in numbers and that internecine strife is the factor ultimately responsible for population decrease. During a five-year period (1965 to 1970) Krebs and his colleagues studied the anatomy of field vole populations (Microtus pennsylvanicus and M.ochrogaster) in southern Indiana. The mechanics of the decline were obvious -a reduction in survival of early juveniles and in birth rate, but more important an increased mortality among the older animals. This increased mortality affected males and females differently at different times of the year. That mortality acts selectively in this way is thought to demonstrate that extrinsic factors are not the chief forces in population decline.

In order to investigate the intrinsic quality of population decline, Krebs et al. built a Microtus-proof enclosure and compared population increase within the fence with a control area outside it.
The number of animals in both groups increased sharply but became excessively high only within the enclosure. It scemed that prevention of dispersal was largely the root cause. In the adjacent control area, the percentage losses of voles as a result of dispersal were much higher during population increase than during peak densities of population decline.

Using the genes $T f$ (transferrin) and $L A P$ (leucine aminopeptidase) as markers, the authors found at these two loci large changes in gene frequencies which could be related to dynamic change in population number. During the increase phase, 89 per cent of the losses of heterozygous females $\left(T f^{c} / T f^{c}-c\right.$ and $e$ are alleles of the $T f$ gene) from the control populations were caused by dispersal. This suggests that demographic events are genetically selective and dispersal losses are not equal over all genotypes. This is the first time a genotype for dispersal has been demonstrated in a natural population.

The hypotheses of population regulation by social stress have to explain the finding of Microtus populations in the enclosures at densities many times higher than normal without "stress" taking an obvious toll. Prevention of dispersal changes the quality of the enclosed populations compared with that of the controls. In spite of the high density they continue to breed, but the behavioural effects of crowding do not seem to cause voles to die. Only when starvation takes effect by decreasing survival and reproduction does the rate of increase start to drop off. However productive they may be, fencing experiments turn up many new questions. For example, does the differential dispersal rate of animals during the increase phase of the cycle cause the quality of voles remaining at peak densities in the wild to be different from the quality observed at far higher densities in enclosures?

Several experiments are suggested by Krebs et al. to examine the components and influences of dispersal. The first is to attempt to cause abnormal densities in other rodent species by preventing dispersal. Second, one-way doors fitted to fenced enclosures should allow population regulation through dispersal to occur. Third, because dispersal is relatively unimportant once peak density has been achieved, closing the doors at this time should have no effect on normal decline. Krebs et al. themselves state that they do not know whether the genetic changes they describe are causally related to population changes, or are just side effects of other changes.

The ideas reported here naturally refer to existent populations of voles. Dispersers from increasing populations colonize unoccupied areas and, because of the increased incidence of heterozygotes among their number, will establish populations rich in $T f$ heterozygotes. This does not seem to bestow any observable ecological fitness at this stage in the demographic pattern. Evidence from island studies indicates that isolated rodent populations are much like land-locked populations in their demographic development. In the light of the new observations it is these populations founded by colonizers from other populations, and in which dispersal is largely prevented, that should now be closely scrutinized.

\title{
Cyclic AMP and Chloramphenicol Acelyl Transferase
}

The antibiotic inactivating enzyme chloramphenicol acetyl transferase is one of the numerous enzymes in Escherichia coli the synthesis of which seems to be regulated by cyclic AMP, but unlike the inducible enzymes involved in the metabolism of sugars, which are also regulated by cyclic AMP, chloramphenicol acetyl transferase is made constitutively. How cyclic AMP regulates the synthesis of chloramphenicol acetyl transferase is the question which de Crombrugghe, Paston, Shaw and Rosner have been investigating. As they report in Nature New Biology next week (February 21) it seems that both cyclic AMP and the nucleoside tetraphosphate ppGpp independently stimulate the synthesis of this enzyme.

The group used an ingenious and very sensitive assay for chloramphenicol acetyl transferase and found that cyclic AMP stimulates its synthesis about twofold. This indicates that the synthesis of this enzyme is not as strictly con- trolled by cyclic AMP as is the synthesis of $\beta$-galactosidase. To investigate the control mechanism further, de Crombrugghe et al. set up a cell-free system which synthesizes chloramphenicol acetyl transferase and responds to cyclic AMP which then stimulates a fifteenfold increase in the synthesis of this enzyme. This stimulation is specific, but ppGpp in the absence of cyclic AMP also enhances synthesis of the enzyme. By contrast, ppGpp alone does not stimulate synthesis of enzymes of the lac operon and other inducible enzymes. In short, the synthesis of chloramphenicol acetyl transferase is enhanced by cyclic AMP and ppGpp alone, whereas synthesis of other $E$. coli enzymes by ppGpp depends on the presence of cyclic AMP.

These differences justify further experiments aimed at elucidating in detail the mechanism of regulation of synthesis of this antibiotic inactivating enzyme. 